\title{
Coulisses
}

Revue de théâtre

\section{Les didascalies de Divinas palabras de Ramón del Valle-Inclán}

«Scènes et cris »

\section{Samantha Faubert}

\section{CpenEdition}

\section{Journals}

Édition électronique

URL : https://journals.openedition.org/coulisses/974

DOI : $10.4000 /$ coulisses. 974

ISSN : 2546-9460

\section{Éditeur}

Presses universitaires de Franche-Comté

Édition imprimée

Date de publication : 31 décembre 2009

Pagination : 71-86

ISBN : 978-2-84867-270-0

ISSN : 1150-594X

\section{Référence électronique}

Samantha Faubert, «Les didascalies de Divinas palabras de Ramón del Valle-Inclán », Coulisses [En ligne], 39 | Automne 2009, mis en ligne le 30 novembre 2016, consulté le 29 décembre 2022. URL : http://journals.openedition.org/coulisses/974 ; DOI : https://doi.org/10.4000/coulisses.974

\section{Ce document a été généré automatiquement le 29 décembre 2022.}

Tous droits réservés 


\title{
Les didascalies de Divinas palabras de Ramón del Valle-Inclán
}

\author{
«Scènes et cris »
}

Samantha Faubert

1 La problématique principale de l'œuvre dramatique de cet auteur espagnol, né en 1866 et mort en 1936, est celle de l'adaptabilité de ses pièces à la scène. Une importante bibliographie est consacrée à cette question, depuis les articles parus dans la presse espagnole à l'époque de l'auteur jusqu'aux analyses philologiques actuelles. ValleInclán a lui même favorisé cette polémique en répondant par exemple en 1927 au journal $L a$ Voz, qui lui demandait de participer à une enquête sur les dramaturges, qu'il le ferait s'il était auteur dramatique :

Sans doute m'avez-vous placé dans cette catégorie parce que j'ai écrit des œuvres

dialoguées. Mais vous remarquerez que je les ai toujours publiées avec des indications scéniques qui suffisent à les expliquer pour la lecture, sans qu'un histrion intervienne. Si une de mes œuvres a été représentée, j'ai attaché si peu d'importance à l'événement que je n'ai jamais cru devoir rappeler un si lamentable incident, en indiquant dans l'édition la distribution des comédiens ou la date de la représentation. Je me déclare donc totalement étranger au théâtre ${ }^{1}$.

2 La thèse la plus couramment développée pour expliquer pourquoi ce dramaturge s'est rapidement détourné de la scène est celle d'une l'inadéquation entre la scène espagnole du moment et les pièces de Valle-Inclán. En Espagne, la fin du xix et le début du $\mathrm{xx}^{\mathrm{e}}$ siècle est l'époque du théâtre réaliste qui utilise les salons bourgeois, une trame bien ordonnée et un langage conventionnel pour évoquer des conflits sentimentaux ou familiaux. Valle-Inclán avait une conception de la mise en scène toute différente, influencée par le symbolisme ou le "Théâtre d'Art »². Las de la confusion entre vie et art, ces artistes proposent un théâtre d'imagination et de fantaisie où triomphent la poésie, la musique, l'appel des sens, l'intuition, l'irrationnel et les grands espaces. C'est dans cette perspective que Valle-Inclán se réfère au travail du metteur en scène d'origine autrichienne Max Reinhardt (1873-1943) :

En Allemagne, il y a peu de temps, un célèbre metteur en scène - Reinhar (sic) - a mis en scène Le grand théâtre du monde. L'œuvre de Calderón a reçu les plus grands 
éloges non seulement pour ses mérites littéraires mais également pour l'art et le nouveau style de la représentation. (...) La plastique des costumes, les mouvements et les couleurs sont dans le théâtre moderne les miracles de la lumière. ${ }^{3}$ ses œuvres et la scène, l'auteur ait cessé de s'intéresser à la question de la mise en scène et se soit senti plus libre d'écrire ses pièces, en dehors de toutes contraintes. Lorsqu'il écrit Divinas Palabras en 1918-1919, la rupture est consommée et, s'il a imaginé une représentation de sa pièce en l'écrivant, c'est une image mentale idéale qui n'est selon lui accessible qu'au lecteur : "La représentation a-t-elle quelque chose à voir avec les indications scéniques que j'écris? Je suis sûr que mes indications donneront une idée de ce que j'ai voulu faire, beaucoup plus aboutie qu'une représentation. $»^{4}$

Si la question du rapport entre la scène et l'écriture chez Valle-Inclán renvoie à tout son concept scénique, c'est-à-dire, aussi bien à l'analyse des dialogues que des didascalies, il est bien évident que l'étude de ces dernières est un élément central de la problématique. Nous ne souhaitons pas revenir ici sur la pertinence des affirmations d'irreprésentabilité du théâtre de Valle-Inclán - dont l'argumentation s'est le plus souvent basée sur la nature poétique de ses didascalies. Notre réflexion ne prendra pas non plus parti dans le débat concernant l'aspect injonctif et utilitaire des didascalies, perçues uniquement dans la perspective de la scène et comme des textes dont le destinataire est avant tout le metteur en scène. ${ }^{5}$ Cette conception amène à opposer écriture didascalique et mise en scène, ce qui n'est évidemment pas le propos de ValleInclán.

L'écriture didascalique dans le théâtre moderne est donc le lieu d'un conflit qui informe la nature du texte, le lieu d'un tiraillement entre une subjectivité réelle qui utilise le langage comme arme et une subjectivité virtuelle qui agira en dernier lieu. Le paratexte n'est donc ni un texte à côté ni un texte autour, mais un texte contre le texte de la représentation, sans lequel il ne pourrait pas cependant exister. ${ }^{6}$

Les pièces de Valle-Inclán semblent plutôt être un ensemble tout entier destiné au lecteur, dans lequel dialogues et didascalies se répondent, se complètent, s'interpénètrent pour former conjointement la fiction, le poème. C'est ce poème qui pourra donner lieu à la représentation, par un jeu de communication entre les arts cher à Valle-Inclán.

6 Les didascalies valleinclanesques, bien que pouvant être qualifiées d'inhabituelles, conservent la plupart du temps leur fonction dramatique traditionnelle, c'est-à-dire qu'elles présentent les personnages, introduisent leurs paroles, renseignent sur la façon dont celles-ci doivent être prononcées, décrivent l'espace, apportent des indications temporelles et organisent la macrostructure de la pièce ${ }^{7}$. L'originalité de ce dramaturge réside dans la manière dont ses didascalies remplissent ces fonctions. Notre analyse relèvera donc certains aspects particulièrement pertinents de l'étude des didascalies de Divinas Palabras, en les mettant sans cesse en perspective avec une pratique plus traditionnelle de l'écriture didascalique et surtout dans l'objectif de mettre en évidence le concept scénique de l'auteur.

\section{Comme un roman}

7 On a souvent relevé le caractère narratif du texte didascalique valleinclanesque. D'autres dramaturges ont depuis pris l'habitude de narrativiser les macro-didascalies 
d'ouverture (qui constituent ce que Sanda Golopentia appelle «le prélude didascalique $\left.»^{8}\right)$. Il est vrai que, chez Valle-Inclán, les didascalies narratives occupent parfois tout l'espace didascalique et non seulement le début de la pièce ou de chaque acte. Mais pourquoi reprocher leur aspect continu, logique, explicatif et descriptif à ces paragraphes destinés à donner un contexte aux discours des personnages? Il nous semble que l'important n'est pas la question d'un style davantage adapté au roman mais bien le fait que la narration semble obstruer l'échange théâtral, par une présence forte de l'auteur qui s'impose entre personnages et lecteur. Ce chapitre prétend donc étudier, au-delà de l'aspect narratif, cette relation auteur/lecteur qui n'est pas le rapport dramaturgique traditionnel et qui est l'instrument d'un concept théâtral propre.

Les dramatis personae tiennent une place privilégiée dans l'instauration d'un pacte de lecture. Dès les premières pages du livre, la liste des personnages vise à orienter le lecteur parmi l'ensemble des protagonistes de la pièce qui, dans Divinas Palabras, sont nombreux. Valle-Inclán crée une écriture dramatique propre dès les dramatis personae. Le texte échappe parfois à l'énumération neutre par un jeu sur la ponctuation ou des tentatives de construction grammaticale ( Pedro Gailo, sacristán de San Clemente; Mari-Gaila, su mujer, y Simoniña, nacida de los dos. $\left.»^{9}\right)$, ou par l'insertion d'éléments hétéroclites au milieux des personnages: groupes de personnes («Mujerucas que llenan los cántaros en la fuente. ", «Marica del Reino con otras mujerucas. $\left.»^{10}\right)$, animaux ( Un sapo anónimo que canta en la noche. $\left.»^{11}\right)$, cris ( Final de gritos y atrujos moceriles $\left.»^{12}\right)$.

Ces éléments constitutifs des dramatis personae valleinclanesques ont déjà été relevés mais on a moins remarqué la fonction remplie par certaines didascalies internes qui complètent les dramatis personae. Ainsi, une description ou un portrait situés au milieu de la pièce viennent préciser et amplifier le tableau des personnages qui se construit petit à petit, et pas nécessairement là où on l'attendrait. Dans Divinas Palabras, alors que le chien savant Coimbra est décrit dans le détail dix pages après sa première apparition, Colorín, l'oiseau diseur de bonne aventure, n'est mentionné qu'à la p. 131 quand de toute évidence il est présent sur scène depuis l'entrée de son maître p. 115. De même, la description de Ludovina, la tavernière, apparaît, par un effet de dilation, après la didascalie inaugurale de la scène VII et la question que la Tatula lui adresse :

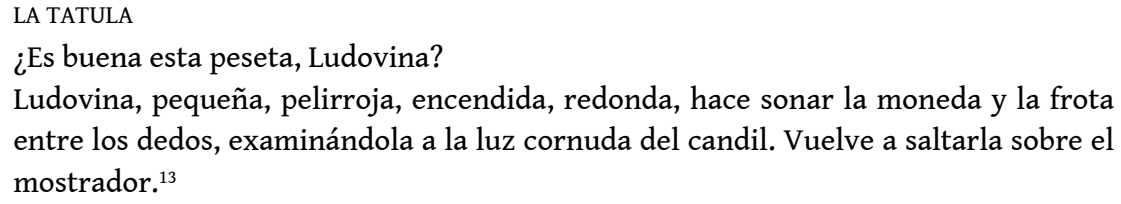

10 La didascalie de début de scène n'a pas joué son rôle d'introduction des personnages, rôle repris après coup par une didascalie intermédiaire, alors que le personnage est déjà convoqué sur la scène. La réplique de la Tatula qui se termine par le nom du personnage «Ludovina » est le déclencheur qui amène la description, procédé narratif propre au roman. Les personnages acquièrent un visage au fur et à mesure de la pièce, on ne peut compter sur les dramatis personae pour tout donner à voir. Ces effets de suspens ou de progressivité dans la perception des personnages seraient peu opérants dans un texte dramatique qui n'aurait d'autre objet que la transposition à la scène. L'auteur n'a de toutes évidences aucune intention de s'effacer derrière une fonction purement utilitaire des indications scéniques. Le visuel se construit de façon fragmentaire, comme un puzzle ou comme un tableau mal éclairé sur lequel les ombres 
se déplacent pour révéler peu à peu des espaces invisibles auparavant. Cette manipulation de l'écriture dramatique contraste avec un genre où tout est donné à voir sur la scène une fois le rideau levé et après l'entrée du personnage. (Ce procédé serait sans doute à rapprocher de l'écriture cinématographique à laquelle Valle-Inclán s'intéressait.) Le dramaturge réussit à construire une image en devenir dans un genre caractérisé par l'ici et maintenant.

11 Les dramatis personae ne sont donc plus ce texte extérieur et antérieur à la pièce dans lequel l'auteur donne, une fois pour toute et avant l'action, toutes les connaissances générales sur les personnages. Elles n'ont plus le rôle de préparer la lecture car la perception des personnages se construit dans un rapport dialectique entre dramatis personae et didascalies internes. Dans Divinas Palabras, on assiste a une dilution des fonctions didascaliques traditionnelles au profit d'une homogénéité du texte (poème): échos ou jeux de questions-réponses d'une didascalie à l'autre et entre répliques et didascalies. La hiérarchie théâtrale est profondément bouleversée.

Dans cette refonte, l'indication qui est censée introduire ou commander une réplique peut avoir au contraire à la reprendre, à la préciser après coup, lui conférant par là même une autre dimension. Ainsi, la fonction préparatoire des dramatis personae se retrouve dans d'autres types de didascalies comme celles qui visent à décrire la façon de parler des personnages et que Sanda Golopentia appel «didascalies locutoires ${ }^{14}$. Comme leur fonction l'indique, ces informations doivent intervenir avant la réplique afin de conformer la diction du personnage-acteur. Nous avons trouvé un cas dans Divinas Palabras où l'ordre est inversé. L'écriture dramatique s'en trouve ralentie et l'aspect contemplatif de la didascalie dû à son caractère descriptif et poétique est renforcé. Après la sombre déclamation de Marica del Reino qui prédit une fin funeste à son frère, l'effet rétroactif de la didascalie donne un aspect encore plus solennel à la scène :

\section{MARICA DEL REINO}

¡En qué hora triste fuiste nacido! ¡Jamás de los jamases me quitaré el luto de encima, si llevas a cabo tu mal pensamiento! ¡Ay, hermano mío, antes quisiera verte entre cuatro velas que sacando filo al cuchillo! ¡Celos con rabia a la puerta de la casa, nunca dictaron buen consejo! ¡Ay, hermano mío, sentenciado sin remedio! ¡Cuando quieres mirar por tu honra, te echas encima una cadena! ¡Esconde el cuchillo, hermano mío, no le saques filo! ¡No te comprometas, que solamente de considerarlo, toda el alma se me enciende contra esta mala mujer! ¡La gran Anabolena se desvaneció con el carretón! ¡Ay, hermano mío! ¿Por qué es tan tirana la honra, que te ordena cachear, en busca de esa mujer, hasta los profundos de la tierra?

Las voces declamadoras de aquella vieja, en el silencio del atrio lleno de sombras moradas, de fragancias de rocío, de vuelos inocentes de pájaros, tienen el sentido de las negras sugestiones, en la primera inocencia sagrada. ${ }^{15}$

Il nous semble que si cette didascalie avait été placée avant la réplique, elle aurait orienté l'interprétation du lecteur vers une sincérité du personnage. Au lieu de cela, l'effet produit est la saisie de l'intention manipulatrice de la sœur qui, sous couvert de s'inquiéter pour son frère, l'incite au meurtre. La motivation profonde de Marica del Reino transparaît à la fin de la réplique lorsqu'elle mentionne le chariot du nain ("carretón »), objet de convoitise car il est très efficace pour la mendicité. Non seulement la didascalie locutoire rétroactive agit sur le rythme par un effet de pause insistante, mais on peut également relever un contraste entre la violence et la malveillance de la réplique d'une part et certains éléments caractérisant l'ambiance 
dans la didascalie d'autre part : termes mystiques ou religieux («atrio », "sagrada ", couleur pourpre "moradas»), champ lexical de la nature idéalisée ("rocío", «inocentes pájaros»), répétition de la notion d'innocence («inocentes", « inocencia»).

Tout cela tend à construire le concept scénique valleinclanesque relevé par Begoña Riesgo qui unit hétérogénéité et homogénéité. Homogénéisation par la construction progressive des images et hétérogénéité par l'alliance des contraires.

Un créateur supérieur à ses créatures, qui contemple de loin pour mieux appréhender l'essence des choses et en exprimer l'unité - l'image obtenue prend alors la forme d'une totalité homogène et correspond à la figuration d'un ordre divin harmonique dont le poète a l'intuition et qu'il chante lyriquement (les éléments ne s'opposent pas, ils se complètent comme les pièces d'un grand puzzle) - ou bien qui contemple avec ironie et même cruauté - l'image est alors celle d'une totalité hétérogène dont les éléments se répondent mais pour mieux se détruire les uns les autres, c'est sur ce décalage oxymorique que se fonde l'écriture de l'esperpento. ${ }^{16}$

15 L'image du créateur omniscient est particulièrement évidente dans l'écriture didascalique de Valle-Inclán qui n'est plus seulement prescriptive mais surtout interprétative. Elle vise également un espace-temps situé en dehors de l'action de la pièce. Il est ainsi intéressant de remarquer l'invasion des indications scéniques par les références à un autre temps que celui de la pièce: temps itératif de l'habitude, des connaissances universelles ou du passé. Les personnages sont présentés par leurs activités coutumières, ce qui tend à les définir par leur fonction dans la pièce, comme les rouages d'une machinerie, car ce n'est pas la psychologie des personnages qui intéresse Valle-Inclán mais la mécanique des situations et des actions au service d'un projet global.

Es Miguelín el Padronés, uno que anda caminos, al cual por sus dengues le suele acontecer en ferias y mercados que lo corran y afrenten. ${ }^{17}$

16 Ce type de présentation des personnages alterne avec des remarques d'ordre universel posant des connaissances ou proposant une interprétation que seul le didascale ${ }^{18}$ omniscient peut apporter.

La niña, extática, parece una figura de cera entre aquellos dos viejos de retablo, con las arrugas bien dibujadas y los rastros de un ocre caliente y melado, como los patores de una Adoración $»^{19}$

17 Cette didascalie, tout comme celle qui précédemment se rapportait à Marica del Reino, illustre bien comment concept scénique et création esthétique se rejoignent. Par ailleurs, la notion de connaissance universelle est à rapprocher de l'usage de l'article défini relevé par Christian Boix dans les Comedias bárbaras. ${ }^{20}$ Nous en avons trouvé quelques occurrence dans Divinas Palabras, comme cette première mention de la présence des guardias :

La pareja de tricornios, negra y polvorienta, penetra en las sombras del soto donde sestea la taifa de hampones. ${ }^{21}$

L'utilisation du pronom possessif ( su grito $»^{22}$, «su relincho $»^{23}$ ) oriente la réception dans la même direction en comptant que le lecteur établit une relation évidente et obligatoire entre celui qui pousse le cri et le cri lui-même. Le didascale part du principe que tout le monde sait que le nain crie et que le diable-bouc hennit (ou crie). Quant au passé, l'abondance des verbes à l'imparfait, plus que s'y référer, le rend effectif et opératoire dans la pièce. 
El enano había tenido el último temblor. Sus manos infantiles, de cera oscura, se enclavijaban sobre la colcha de remiendos, y la enorme cabeza azulenca, con la lengua entre los labios y los ojos vidriados, parecía degollada. Las moscas del ganado acudían a picar en ella. Ludovina había dejado el mostrador. ${ }^{24}$ didascalique et propose de nouveaux procédés dramaturgiques qui font des indications scéniques l'autre lieu où se construit le dialogue. S'ouvre alors au lecteur un nouvel espace théâtral. Encore une fois, la question n'est pas de savoir si ces procédés sont propres au roman ou si le texte ainsi produit est représentable ${ }^{27}$.

Palabras. Il s'agit de fonds sonores ou de paroles devant être prononcées par un groupe de personnes et dont seule la thématique générale est indiquée : murmures, cris humains ou d'animaux, chants, contes et légendes racontés, dialogues du quotidien, apostrophes, prières, voix de la kermesse (diseurs de bonne aventure), paroles prononcées dans le sommeil, gémissements, bruits de foule. L'abondance (16 occurrences) et la diversité des ces indications témoignent de l'importante fonction qu'elles remplissent et du rôle qu'elles jouent dans le concept scénique. Les cris sont omniprésents dans les répliques, ils sont cités dans les dramatis personae parmi les personnages et Valle-Inclán les mentionne comme éléments définitoires de son théâtre.

À un théâtre qui se fonde sur un concept intellectuel, il oppose un théâtre qui se fonde sur un concept scénique qui consiste à dire les choses en les mettant en scène au sens littéral du mot, c'est-à-dire en les mettant en espace, en formes et en rythme, un langage qui donne à voir et à entendre, qui matérialise par l'image et par le son et que Valle résume dans une formule concise : « escenarios y gritos $»{ }^{28}$

Ainsi, les didascalies forment un tout avec les répliques pour la création d'un espace homogène de la parole. Il n'y a pas de contradiction entre l'aspect poétique des didascalies et la place centrale de la parole dans le théâtre de Valle-Inclán (il a toujours répété que le dialogue était son mode d'expression privilégié). Le système des relations 
qui se nouent entre indications scéniques et répliques est la trame de ce théâtre qui prétend dire le visuel et faire voir le sonore. Les didascalies ne sont plus là seulement pour préciser la manière dont les répliques devront être prononcées (didascalies locutoires) mais également pour donner la parole, pour la rendre possible. C'est ainsi que notre attention est attirée par un certain nombre de verbes de locution. Ceux-ci sont normalement inutiles au théâtre s'ils n'ont pas la fonction d'orienter la réception vers un sentiment ou un état du locuteur. Les didascalies de Divinas Palabras agissent comme si elles étaient des embrayeurs de paroles obligatoires, comme si les répliques ne pouvaient se suffire à elles-mêmes ou comme si le travail sur la matérialité du langage (le travail poétique) se situait surtout dans les actes de paroles. Cela va à l'encontre de la qualification de contemplative attribuée généralement à ces didascalies esthétiques. Le travail sur la locution (la parole, la voix) est central.

Bastián de Condás, alcalde pedáneo, pone guardas a la muerta, y da sus órdenes con una mano en el aire, como si fuese a bendecir.

EL PEDÁNEO

Vosotros, rapaces, aquí firmes, sin desviaros del pie de la finada difunta. ${ }^{29}$

Una vieja comienza un cuento, y el idiota, balanceando la cabeza enorme sobre la almohada de paja, da su grito en la humedad del cementerio.

EL IDIOTA

¡Hou! ¡Hou! ${ }^{30}$

Dans ces deux scènes, la mention de l'acte de locution dans les didascalies est redondante et inopératoire. La répétition de l'acte de parole amplifie le motif. La raison de ces phénomènes d'insistance est à chercher ailleurs que dans la fonction locutoire. Dans les tableaux didascaliques, les verbes ou expressions de locution ne sont pas seulement là pour décrire le locuteur et le contexte de locution. Car la parole renvoie avant tout à elle-même - que l'on songe au titre de la pièce - elle est posée comme un faire et non seulement comme un dire.

Le système théâtral construit par le dramaturge semble parfois se refermer sur luimême. L'écriture est bousculée, le texte piétine sur place quand par endroit la didascalie se fait explicative (comme le souligne le «por que » de l'exemple suivant) :

Un chalán que conduce novillos del monte, levantándose sobre los estribos, da voces por que se aparte del camino.

EL CHALÁN

¡Eh!... ¡No me espantes el ganado! ${ }^{31}$

La vieja, raída y pelona, saca la cabeza por el ventano, y con gritos espanta a las bestias.

MARICA DEL REINO

¡Cache!... ¡Cache!... ¡Cache, grandísimos ladrones!... ${ }^{32}$

A d'autres moments, c'est la répétition mot pour mot (entre la didascalie intermédiaire et l'indication du nom du groupe de personnages) qui manifeste ce piétinement :

En torno de la casa vuelve a rodar la copla de los rapaces.

COPLA DE RAPACES

¡Tunturuntún! La Mari-Gaila.

¡Tunturuntún! No sé qué le dio. 
¡Tunturuntún! La Mari-Gaila.

¡Tunturuntún! Que malparió. ${ }^{33}$ mentions. Et la didascalie reprend ce que la réplique a déjà dit, comme dans la dernière scène de la deuxième journée où, après avoir ponctué les «Jujurujú » du diable-bouc par des « lanza su relincho $»^{34}$ ou « deja oír su relincho $»^{35}$ introductifs, une didascalie se fait écho postérieur du cri en ajoutant : «El Cabrío revienta en una risada $»^{36}$. Ce cas n'est pas isolé, il arrive parfois qu'on ait l'impression que le didascale réagit après coup aux paroles des personnages, comme dans cet exemple où la didascalie répète les mots de la réplique, reprenant le surnom attribué par le personnage à Marica del Reino :

UNA MOZA

¡Conformidad, tía Marica!

Tía Marica, ayudada por las mujeres y cubierta con el manteo, camina encorvada. ${ }^{37}$

En remettant en cause la distinction des rôles entre didascalies et répliques dans le texte théâtral, Valle-Inclán montre que la parole est partout. C'est bien le sens de la dernière scène. Les mots accèdent au statut suprême lorsqu'ils sont en latin, ils sont le dernier refuge, ils donnent à la vie son rythme et son souffle :

¡Milagros del latín! Una emoción religiosa y litúrgica conmueve las conciencias, y cambia el sangriento resplandor de los rostros. Las viejas almas infantiles respiran su aroma de vida eterna. $^{38}$

Mari-Gaila, armoniosa y desnuda, pisando descalza sobre las piedras sepulcrales, percibe el ritmo de la vida bajo un velo de lágrimas. Al penetrar en la sombra del pórtico, la enorme cabeza del idiota, coronada de camelias, se le aparece como una cabeza de ángel. Conducida de la mano del marido, la mujer adúltera se acoge al asilo de la iglesia, circundada del áureo y religioso prestigio, que en aquel mundo milagrero, de almas rudas, intuye el latín ignoto de las DIVINAS PALABRAS ${ }^{39}$

La nature de la parole est alors remise en question. Dans les didascalies, elle n'est plus ce texte logique, rationnel et pratique qui prépare la mise en scène. Car le sens de l'écriture valleinclanesque passe avant tout par la matérialité du langage. La poésie du texte didascalique fonctionne sur une manipulation du langage à plusieurs niveaux : tout d'abord l'invention d'expressions ${ }^{40}$, le choix du vocabulaire. Ainsi, le chariot du nain est-il fréquemment désigné dans les didascalies par "dornajo », terme rare ou régional (du galicien «dornallo») qui fait référence à une auge à cochons ou à une grande bassine, alors que les personnages emploient uniquement le mot « carretón ». Il arrive aussi que le mot acquière un sens légèrement différent du sens commun. Cette amplification sémantique, le dramaturge l'explique dans La Lámpara maravillosa, à propos de la signification personnelle qu'il donne à certains vocables: "j'aspire à l'exprimer en donnant aux mots par dessus la valeur que tous leur accordent, une valeur émotive engendrée par moi. » ${ }^{41} \mathrm{Et}$ il cite Verlaine : "Choisis tes mots toujours en te trompant un peu. $»^{42}$

31 Nous avons déjà relevé l'importance du rythme dans l'écriture de Valle-Inclán. Il affectionne particulièrement les groupes de deux ou trois adjectifs. Begoña Riesgo Demange a souligné l'apparentement du style du dramaturge avec la musique et l'opéra ${ }^{43}$, et les rythmes binaires et ternaires montrent effectivement une construction du texte comme une partition musicale. 
La madre blanca y rubia, risueña de ojos, armónica en los ritmos del cuerpo y de la voz. La hija abobada, lechosa, redonda con algo de luna, de vaca y de pan. ${ }^{44}$

"Rítmica y antigua, adusta y resuelta, [Mari-Gaila] levanta su blanca desnudez ante el río cubierto de oros. ${ }^{45}$ non seulement le rythme mais également les échos d'une scène à l'autre que représentent les nombreuses répétitions. Leur abondance et le choix de leur place témoignent de leur participation au système esthétique construit par les didascalies. Appliqués à la description des personnages, les mots répétés sont à la fois un point de repère qui permet la reconnaissance visuelle du protagoniste et également un procédé de caricature des êtres qui vont se résumer à quelques éléments réducteurs. Ainsi, Pedro Gailo est noir, Mari-Gaila est rythmique et souriante, Simoniña blanche et ronde (les même adjectifs s'appliquent d'ailleurs à la lune $\left.{ }^{46}\right)$... On peut également lire deux fois le même syntagme nominal dans les didascalies internes d'une même scène, à quelques pages d'intervalle.

La Mari-Gaila, gozosa de su nueva ventura, sofocada y risueña, llega tirando del dornajo, por la carretera cegadora. ${ }^{47}$

El correaje, los fusiles, los tricornios, destellan en la carretera cegadora de luz. ${ }^{48}$

La répétition n'est pas un procédé littéraire au service d'un effet, c'est un des principes créateurs de l'écriture de Valle-Inclán. Certains mots envahissent l'œuvre, tant on les retrouve déclinés tout au long de la pièce. Nous avons ainsi pu relever un important réseau signifiant constitué par le terme " cera » (au moins 10 occurrences). "Cera » ne désigne pas un objet clé dans l'histoire mais s'emploie uniquement comme élément de comparaison dans des descriptions, pour manifester la présence de la mort : trois fois à propos de Marica del Reino avant et après sa mort ${ }^{49}$; deux fois pour décrire la fillette aux vieillards dont la caractéristique principale est son aspect statique et irréel ${ }^{50}$; quatre fois en référence au cadavre du nain ${ }^{51}$.

Enfin, les couleurs, les odeurs ou les parfums, alliés aux éléments auditifs, ponctuent le poème didascalique fondé sur un langage des sens. Tout est dit, senti et montré à la fois.

San Clemente. La iglesia románica de piedras doradas. La quintana verde. Paz y aromas. El sol traza sus juveniles caminos de ensueño sobre la esmeralda del río. ${ }^{52}$

Ainsi, dialogues et didascalies fonctionnent ensemble pour élaborer un théâtre-poème synthétique où palpite la nature.

Le texte didascalique devient donc ce poème convoquant tous les sens qui donnera lieu à une représentation synthétisant les arts. C'est le cœur du concept scénique de Valle-Inclán. Si Divinas Palabras interroge la création littéraire dans ses fondements en questionnant le rôle de la parole, c'est tout le système théâtral de l'auteur qui repense la relation au verbe. Le concept scénique de Valle-Inclán ne se résume pas aux didascalies mais elles y jouent un rôle prépondérant car elles sont le lieu d'une plus grande manipulation de l'écriture dramatique. Au-delà de son théâtre, toute l'œuvre de cet auteur tend à repenser la littérature dans ses implications universelles. Valle-Inclán exprime par là sa sensation de n'être que le maillon d'un grand poème symphonique 
inaccessible que l'on entrevoit parfois à travers l'homogénéité de son système théâtral et les sonorités qui se répondent en écho dans ses didascalies.

\section{NOTES}

1. La Voz, 20 mai 1927, dans Dougherty, Dru, Un Valle-Inclán olvidado : entrevistas y conferencias, Madrid, Fundamentos, 1983, p.164. La traduction est de nous.

2. Le terme "théâtre d'art " apparaît pour la première fois en 1890, désignant le théâtre fondé par Paul Fort. Dès le début du siècle, il est successivement employé dans divers pays d'Europe : Stanislavski et Nemirovitch-Dantchenko fondent le Théâtre d'Art de Moscou, Fritz Erler et Georg Fuchs celui de Munich, tandis que Craig élabore la théorie d'un théâtre d'art idéal. Cette notion va très vite représenter un véritable programme qui tend à rehausser le niveau artistique du théâtre jusqu'à lui conférer un statut spécifique parmi les autres arts.

3. Lettre à Juan José Santa Cruz datée du 8 janvier 1926, parue dans $A B C, 26$ février 1988, p. 47. La traduction est de nous. La mise en scène de Calderón par Reinhardt à laquelle il est fait allusion eut lieu à Salzbourg en 1922. La traduction est de nous.

4. Paroles de Valle-Inclán à sa femme et transcrite par elle dans une lettre à Azaña datée de 1936. Publiée par SCHIAVO, Leda dans «Cartas inéditas de Valle-Inclán I », Insula, n 398, janvier 1980, p.10. La traduction est de nous.

5. Lire Patrice Pavis et Anne Ubersfeld.

6. Martinez Thomas, Monique. «L'influence de Valle-Inclán sur la dramaturgie contemporaine ", in Livre et édition dans le monde hispanique. XVIe-XXe siècles. Pratiques et discours paratextuels, Actes du colloque international, Grenoble 14-16 novembre 1991, Grenoble, Université Stendhal, 1992, p. 231.

7. Cette caractéristique des didascalies de Valle-Inclán a été relevée par Monique Martinez Thomas dans Voir les didascalies. Ibéricas, n³, 1994, Sanda Golopentia et Monique Martinez Thomas ed., p. 223 : «Les texte didascalique valleinclanesque, même en vers, nous semble donc donner des ordres précis pour la mise en scène. Le mode d'énonciation choisi par l'auteur de théâtre, s'il semble privilégier le lecteur, est créateur de sens pour une future action scénique. Il n'y a pas renoncement à la mise en scène dans la mesure où nous retrouvons tous les éléments d'un système didascalique complet par rapport à la représentation. »

8. Golopentia, Sanda, ibid.

9. Ramón del Valle-Inclan, Divinas Palabras. Tragicomedia de aldea, Edición crítica de Luis Iglesias Feijoo, Espasa Calpe, 1991, p.109. Toutes les citations de la pièce sont tirées de cette édition.

«Pedro Gailo, sacristain de San Clemente; Mari-Gaila, sa femme et Simoniña, enfantée par eux deux. »

Traduction de Jean-Marie Broucaret, Actes Sud 1989, p. 10. Toutes les traductions sont tirées de cette édition.

10. p. 110.

«Commères, qui remplissent les cruches à la fontaine. ", p. 10.

11. p. 111.

"Un crapaud, anonyme, qui chante dans la nuit. », p. 10.

12. Ibid.

« Final de cris et de sarabandes juvéniles. », p. 10. 
13. p. 282.

« ROSA LA TATULA. Elle est bonne cette peseta, Ludovina? », p. 51.

14. Op. cit. p. 118.

15. p. 252-253.

"MARICADELREINO. Ay! Quelle est triste l'heure où tu es né ! Jamais au grand jamais je ne quitterai le deuil si tu exécutes ta mauvaise pensée! Ay! Mon frère, j'aimerais mieux te voir entre quatre cierges plutôt qu'aiguiser le couteau! Jamais la fureur jalouse à la porte de la maison n'a dicté de bons conseils! Ay! Mon frère, condamné sans espoir ! Si tu veux retrouver ton honneur, tu te passes les chaînes! Cache le couteau, frère, ne l'aiguise pas! Ne te compromets pas! Rien que d'y penser mon âme s'enflamme contre cette maudite femme! La grande scélérate s'est évanouie avec le chariot! Ay! Mon frère! Pourquoi l'honneur est si tyrannique qu'il t'ordonne, pour retrouver cette femme, de fouiller jusque dans les profondeurs de la terre?

Les cris déclamatoires de cette vieille, dans le silence du parvis rempli d'ombres violettes, de parfum de rosée, de vols innocents d'oiseaux, évoquent les conseils sataniques aux premiers temps de la sainte innocence. ", p. 43-44.

16. Riesgo Demange, Begoña. "Valle-Inclán : du concept scénique au concept esthétique », Hispanísticas XX, nº15 (Le spectacle au XXème siècle), Université de Bourgogne, 1997, p.91. L'« esperpento » est un genre littéraire créé par Valle-Inclán où la réalité est déformée de manière systématique, avec une insistance de l'écriture sur le grotesque et sur l'absurde.

17. p. 140-141.

"C'est Miguelín el Padronés, un vagabond. A cause de ses mines efféminées, il a l'habitude dans les foires et les marchés d'être pourchassé et outragé. », p. 17.

18. Terme emprunté à Monique Martinez Thomas.

19. p. 288.

"L'enfant extatique paraît une statue de cire entre ces deux vieux de retable, aux rides bien dessinées et aux visages d'un ocre chaud et miellé, comme les bergers d'une Adoration. ", p.52.

20. «La propriété linguistique essentielle de l'article défini, c'est d'actualiser le substantif du point de vue de sa spécificité contextuelle et situationnelle, à l'intérieur d'une classe dont l'existence est présupposée. Alors que l'article indéfini POSE une réalité en jouant un rôle extracteur sémantique, la détermination définie PRESUPPOSE la présentation antérieure de cette réalité. »

Boix, Christian. "Didascalias bárbaras et comédies du même nom ", in Lire Valle-Inclán. Les Comédies barbares, Hispanísticas XX, Jean-Marie LAVAUD ed., Université de Bourgogne, 1996, p. 147. 21. p. 222.

"Une paire de tricornes noirs et poussiéreux pénètrent sous les ombrages du sous-bois où la bande de mendiants fait la sieste. ", p. 36.

22. p. 197.

« son cri », p. 30.

23. p. 304.

« son cri », p. 56.

24. p. 292.

" «Le nain a eu un dernier tremblement. Ses mains d'enfant, de cire noire, sont crispées sur la couverture rapiécée et l'énorme tête bleuie, la langue entre les lèvres et les yeux vitreux, semble tranchée. ", p. 54.

25. «A côté des passés simples qui assurent la progression de l'histoire, les imparfaits marquent les procès qui ne participent pas à cette progression. "

Maingueneau, Dominique. Eléments de linguistique pour le texte littéraire, Paris, Nathan, 2000, p. 58.

26. Meschonnic, Henri. "Le Théâtre dans la voix », La Licorne n 41 (Penser la voix), Université de Poitiers, 1997, p. 39. 
27. Contrairement à ce que Luis Iglesias Feijoo écrit dans l'introduction à son édition critique de Divinas Palabras :

«Ese tipo de acotaciones, que por veces introducen referencias a hechos que debían ser verbalizados y por tanto parecen propios de una novela (« después las criadas hablan de una vaca que, en la montaña, parió un choto con dos cabezas ", concluye una escena de Águila de Blasón), se destinan a la lectura, especialmente a la lectura.»

Op. cit. On peut retrouver la citation tirée de Águila de Blasón dans l'édition: Madrid, Espasa Calpe, 1992, p. 98.

«Ce type d'indications scéniques, qui introduisent parfois des références à des faits qui devraient être verbalisés et qui semblent donc propres au roman («puis les servantes parlent d'une vache qui, dans la montagne, a mis bas un veau à deux têtes.", conclut une scène de l'Aigle emblématique), sont destinées à la lecture, uniquement à la lecture. » La traduction est de nous excepté la citation de l'Aigle emblématique : traduction d'Armando Llamas, Actes Sud, 1991, p. 97.

28. Riesgo Demange, Begoña. Op. cit., p. 80. Elle cite un article de Luis Emilio Soto, «En Torno al teatro ", La Nación, Buenos Aires, 3/3/1929.

Traduction des termes espagnols : "scènes et cris ".

29. p. 167-168.

«Bastián de Candás, l'alcalde pédané, place ses gardes devant la morte, et donne des ordres, la main en l'air, comme s'il était en train de bénir.

L'ALCALDE. Vous, garçon, restez ici sans bouger aux pieds de la défunte. », p. 23.

30. p. 196-197.

"Une vieille commence un conte, et l'idiot, balançant son énorme tête sur l'oreiller de paille, pousse son cri dans l'humidité du cimetière.

L'IDIOT : Hou ! Hou ! », p. 31.

31. p. 135-136.

«Un bouvier, qui conduit un lot de jeunes taureaux sauvages, se soulève sur ses étriers et crie pour que la vieille s'écarte du chemin.

LA BOUVIER. Hé !... Ne fais pas peur à mon troupeau ! », p. 15.

32. p. 322.

"La vieille, sèche, les cheveux rares, sort la tête par la fenêtre et avec des cris chasse les bêtes.

MARICA DEL REINO. Cache !... Cache !... Cache, grands voleurs !... », p. 61.

33. p. 343.

«Autour de la maison la copla des gamins revient tournoyer.

COPLA DES GAMINS.

Tunturuntun! La Mari-Gaila

Tunturuntun! Qu'est-ce qui s'est passé ?

Tunturuntun! La Mari-Gaila

Tunturuntun! Elle vient d'avorter. », p. 67.

34. p. 302.

"lance son cri ", p. 56.

35. p. 304.

«laisse entendre son cri », p. 57.

36. p. 305.

« Le bouc éclate de rire. », p. 57.

37. p. 169.

« UNE JEUNE FILLE. Résignez-vous mère Marica!

Mère Marica, aidée par les femmes et couverte de sa mante, marche courbée. », p. 23.

38. p. 393.

"Miracle du latin! Une émotion religieuse et liturgique ébranle les consciences et change l'éclat sanglant des visages. Les vieilles âmes infantiles reprirent un parfum de vie éternelle. », p. 82. 
39. p. 395.

«Mari-Gaila, harmonieuse et nue, marchant pieds nus sur les pierres tombales, perçoit le rythme de la vie sous un voile de larmes. Au moment de pénétrer dans l'ombre du porche, l'énorme tête de l'idiot couronnée de camélias lui apparaît comme une tête d'ange. Conduite par la main de son mari la femme adultère trouve asile dans l'église, entourée du prestige religieux et doré, qui, dans ce monde crédule, aux âmes rudes, accompagne le latin ignoré des

DIVINES PAROLES », p. 82.

40. « ¿ Zueco en dos plantas, dónde irás que lo veas?», p. 188, que Luis Iglesias Feijoo note comme expression d'apparence proverbiale qu'il ne trouve référencée nulle part et qui doit être par conséquent une invention de Valle-Inclán.

«Un seul sabot pour deux pieds bien loin ne peut aller. », p. 27.

41. Del Valle-Inclan, Ramón. La Lámpara maravillosa, Madrid, Espasa calpe, 1948, p. 61.

42. Ibid., p. 68

43. Op.cit.

44. p. 157.

"La mère, blanche, les yeux riants, harmonieuse du corps et de la voix. La fille, hébétée, laiteuse, ronde avec quelque chose de la lune, de la vache et du pain. ", p. 21.

45. p. 384.

"Antique, rythmée, sombre et résolue, elle dresse sa blanche nudité devant la rivière couverte d'ors. ", p. 79.

46. «La luna grande, redonda y abobada, cae sobre el dornajo donde el enano hace siempre la misma mueca. », p. 307.

« La lune grande, ronde, hébétée, tombe sur le chariot où le nain fait toujours la même grimace. », p. 57.

47. p. 210.

« La Mari-Gaila, joyeuse de sa nouvelle aventure, essoufflée et souriante, arrive en tirant le chariot sur le chemin aveuglant de lumière. ", p. 33.

48. p. 225.

«Les cuirs, les fusils, les tricornes étincellent sur la route aveuglante de lumière. », p. 37.

49. " las canillas y los pies descalzos son de cera», p. 138 (" les jambes et les pieds sont de cire ", p. 16) "Las canillas desnudas salen del refajo como dos cirios de cera.», p. 144 ("Les jambes nues sortent du jupon comme deux cierges de cire », p. 17) - « Los pies descalzos y las canillas del color de la cera. », p. 167 ( Les pieds nus et les jambes couleur de cire», p. 23).

50. "La niña, extática, parece una figura de cera entre aquellos dos viejos de retablo", p. 288 ("L'enfant extatique paraît une statue de cire entre ces deux vieux de retable ", p. 52) - «Con sus hábitos morados y sus manos de cera », p. 289 ( « avec son habit violet et ses mains de cire », p. 53).

51. "Sus manos infantiles, de cera oscura », p. 292 (« ses mains d'enfants, de cire noire », p. 54) - « Las manos infantiles, enclavijadas sobre la cobija, tienen un destello cirial», p. 320 ("Ses mains d'enfant, crispées sur la couverture, ont un reflet de cire ", p. 60)- « coronada de camelias la frente de cera ", p. 357 («le front de cire couronné de camélias», p. 71) - «Simoniña (...) esparce las moscas que comen en la cabeza de cera. », p. 360 («Simoniña (...) chasse les mouches qui se posent sur la tête de cire », p. 72).

52. p. 357.

«San Clemente. L'église romane aux pierres dorées. Le cimetière vert. Paix et arôme. Le soleil trace ces juvéniles chemins de rêve sur l'émeraude de la rivière. ", p. 71. 
INDEX

oeuvrecitee Divinas palabras 